\title{
Punção com agulha de Veress no hipocôndrio esquerdo para a criação do pneumoperitônio: valor diagnóstico das provas de posicionamento da agulha em pacientes não selecionados
}

\author{
Veress needle insertion into the left hypochondrium for creation of \\ pneumoperitoneum: diagnostic value of tests to determine the position of the \\ needle in unselected patients
}

\begin{abstract}
Otávio Monteiro Becker Junior, ACBC-SP'; João Luiz Moreira Coutinho Azevedo,ECBC-SP²; Otávio Cansanção de Azevedo'; Octávio Henrique Mendes Hypóllto'; Susana Abe Miyahira33; Gustavo Peixoto Soares Miguel, TCBC-ES4; Afonso Cesar Cabral GUEDES MACHADO, TCBC-SP5
\end{abstract}

\section{RE S U M O}

\begin{abstract}
Objetivo: Verificar a eficiência da punção com agulha de Veress no hipocôndrio esquerdo, a acurácia dos testes descritos para o correto posicionamento intraperitoneal da ponta da agulha de Veress em população não selecionada. Métodos: Noventa e um pacientes, sem quaisquer critérios de exclusão, consecutivamente agendados para procedimentos videolaparoscópicos, tiveram a parede abdominal puncionada no hipocôndrio esquerdo. Os pacientes receberam anestesia geral e ventilação controlada mecânica segundo o protocolo. Após a punção foram utilizadas cinco provas para testar o posicionamento da ponta da agulha no interior da cavidade peritoneal: prova da aspiração - PA, da resistência à infusão - Pres, da recuperação do líquido infundido - Prec, prova do gotejamento - PG, e a prova da pressão intraperitoneal inicial - PPII. Os resultados foram considerados para cálculo da sensibilidade (S) e da especificidade (E) e valores preditivos positivos (VPP) e valores preditivos negativos (VPN). Métodos inferenciais estatísticos foram utilizados na análise dos achados. Resultados: Ocorreram 13 fracassos. A PA teve $E=100 \%$ e VPN=100\%. Pres teve $S=100 \% ; E=0 ; V P P=85,71 \%$ VPN= não se aplica. Prec: $S=100 \% ; E=53,84 \% ; V P P=92,85 \% ; V P N=100 \%$. $P G: S=100 \% ; E=$ 61,53\%; VPP= 93,97\% VPN=100\%. Na PPII, a S, E, VPP e VPN foram de 100\%. Conclusão: A punção no hipocôndrio esquerdo é eficiente, as provas realizadas orientam o cirurgião a despeito do gênero, IMC ou operações prévias.
\end{abstract}

Descritores: Pneumoperitônio artificial. Biópsia por agulha. Procedimentos cirúrgicos minimamente invasivos. Laparoscopia. Laparoscopia, Efeitos adversos.

\section{INTRODUÇÃO}

A criação do pneumoperitônio é o primeiro passo para a realização da videolaparoscopia. A maioria das complicações associadas a esse procedimento ocorre durante a sua etapa mais crítica, o acesso à cavidade peritoneal ${ }^{1}$, em razão do risco significativo de lesões vasculares e viscerais ${ }^{2}$

Os ferimentos vasculares representam as causas mais comuns de morte em procedimentos laparoscópicos $(15 \text { a } 75 \%)^{3,4}$ seguidos por lesões intestinais despercebidas (25\%). Danos em grandes vasos e nas alças intestinais podem ocorrer quando a agulha de Veress é inserida às cegas no abdome, antes da insuflação, como o que ocorre na técnica fechada ${ }^{3}$. Relatos de processos por erro médico relacionados à videolaparoscopia referem que 18\% das reclamações ocorreram como decorrência de acidentes na produção do pneumoperitônio e calcula-se que cerca da metade de todas as complicações laparoscópicas foi atribuída a problemas técnicos ocorridos nesta fase $e^{4}$.

Apesar de não existir consenso quanto ao meIhor método para o acesso à cavidade peritoneal ${ }^{5}$, a punção com agulha de Veress ${ }^{6}$ é a técnica mais frequentemente utilizada ${ }^{2,7}$. Estudo considerando 155.987 procedimentos laparoscópicos, em $81 \%$ deles foi utilizada a aguIha de Veress ${ }^{7}$.

O local clássico da punção é a linha mediana do abdome, junto à cicatriz umbilical ${ }^{8}$. Nessa região, a pun-

Trabalho realizado no Setor de Cirurgia Minimamente Invasiva da Disciplina de Técnica Operatória e Cirurgia Experimental do Departamento de Cirurgia da Universidade Federal de São Paulo e. Serviço de Gastroenterologia Cirúrgica do Hospital Municipal José de Carvalho Florence, São José dos Campos, São Paulo-BR.

1. Doutor em Ciências pela Universidade Federal de São Paulo (UNIFESP) - SP-BR; 2 Professor Livre-Docente do Departamento de Cirurgia da Universidade Federal de São Paulo (UNIFESP) - SP- BR; 3.Aluna do Curso de Doutorado da Universidade Federal de São Paulo (UNIFESP) - SPBR; 4. Professor Adjunto do Departamento de Cirurgia da Universidade Federal do Espirito Santo - ES-BR; 5. Mestrando da Universidade Federal de São Paulo (UNIFESP) - SP- BR. 
ção apresenta riscos de lesão de grandes vasos, em função da pequena distância da parede anterior do abdome com essas estruturas vasculares retroperitoneais ${ }^{9}$. Em pessoas magras, essa distância pode ser menor que dois centímetros $^{3}$. A aorta abdominal e a veia cava inferior, assim como os vasos ilíacos comuns, são particularmente propensos a ferimentos durante a punção com agulha de Veress, nas proximidades da cicatriz umbilical ${ }^{10}$. Apesar da prevalência dessa ocorrência ser baixa $(0,05 \%$ a $0,5 \%)$, a mortalidade atinge índices entre $8 \%$ e $17 \%$, podendo chegar a $21 \%$ por lesões intestinais desapercebidas ${ }^{4-11}$. A gravidade da ocorrência desse tipo de lesão iatrogênica é minimizada quando as punções são feitas em locais afastados da linha mediana ${ }^{12-15}$

Adicionalmente, pacientes com operação abdominal prévia têm risco aumentado de lesões viscerais por agulha de Veress, por causa de aderências peritoneais que tipicamente ocorrem no nível da cicatrização da incisão cirúrgica no peritônio parietal anterior. Estudos necroscópicos encontraram aderências em 74\% a 95\% de pacientes com intervenções cirúrgicas abdominais prévi$\mathrm{as}^{3}$. As incisões medianas são as que apresentam o maior risco de aderências em torno da cicatriz umbilical. Não obstante, mesmo incisões abdominais afastadas do umbigo podem determinar a formação de aderências na região periumbilical. $^{3}$

A punção no hipocôndrio esquerdo tem sido mencionada como sendo segura, sem risco de lesão iatrogênica maior ${ }^{12-16}$. Ressalte-se que, especificamente na região do hipocôndrio esquerdo, é muito rara a ocorrência de aderências peritoneais na parede abdominal, pois é sabido que os movimentos respiratórios do diafragma mobilizam constantemente as estruturas nessa região e dificultam a adesão das mesmas à parede abdominal anterior. Por isso a punção no hipocôndrio esquerdo é a preferida por alguns cirurgiões, em pacientes com laparotomia prévia $^{13}$. Há também cirurgiões que realizam intervenções cirúrgicas bariátricas e preferem o hipocôndrio esquerdo para a criação do pneumoperitônio em seus pacientes ${ }^{14}$. Tal preferência se deve ao fato de que em pacientes obesos, a técnica aberta apresenta dificuldades adicionais devido ao excesso de peso, e a punção na linha mediana é considerada perigosa devido à espessura do tecido adiposo e da posição alta do umbigo no abdome. Tais características dificultam a punção e podem propiciar lesões, interessando principalmente os grandes vasos retroperitoneais ${ }^{15}$.

Em ensaios clínicos envolvendo pacientes selecionados $^{16-18}$, a eficácia da punção do hipocôndrio esquerdo para a criação do pneumoperitônio artificial foi equivalente a da punção na linha mediana ${ }^{16-17}$ (considerada por muitos a técnica padrão-ouro), e sua segurança foi demonstrada ${ }^{18}$.

Adicionalmente, as provas preconizadas para confirmar a posição intraperitoneal da ponta da agulha de Veress mostraram-se adequadas nessa população específica, com dados demográficos e antropomórficos se- letos $^{16-18}$. De forma semelhante, em relação à amostra estudada naquelas pesquisas ${ }^{16-18}$, ficou demonstrado que a pressão intraperitoneal e os volumes de gás injetados nos diversos momentos pré-determinados da pesquisa, são parâmetros úteis e suficientes para orientar o cirurgião quanto ao correto posicionamento da ponta da aguIha de Veress, nos diversos momentos do decorrer da insuflação ${ }^{16-18}$.

Nessa ordem de idéias, resta estabelecer se na população geral, com características demográficas e antropomórficas diversas, as provas de posicionamento da agulha na punção no hipocôndrio esquerdo possam ser universalmente adotadas como parâmetros necessários e suficientes da segura produção do pneumoperitônio.

Há diferenças sobre o valor inicial da pressão intraperitoneal que indicaria o correto posicionamento, variando entre $5^{20}$ e $8 \mathrm{mmHg}{ }^{16}$, considerando-se que valores superiores indicariam posicionamento errôneo (na parede ou em víscera sólida).

Este estudo teve por objetivo verificar a eficiência na criação de pneumoperitônio mediante punção com agulha de Veress no hipocôndrio esquerdo em amostragem extraída indiscriminadamente da população de pacientes submetidos à videolaparoscopia.

\section{MÉTODOS}

Esta pesquisa foi aprovada pelo Comitê de Ética em Pesquisa da Universidade de Taubaté ( $\left.n^{\circ} 0039 / 07\right)$ e pelo Comitê de Ética em pesquisa da Universidade Federal de São Paulo (n¹310/07). Todos os pacientes assinaram termo de consentimento livre e esclarecido.

Noventa e um pacientes, sem quaisquer critérios de exclusão, consecutivamente agendados para serem submetidos a procedimentos videolaparoscópicos no Serviço de Cirurgia Geral do Hospital Municipal José de CarvaIho Florence tiveram a parede abdominal puncionada no hipocôndrio esquerdo, com agulha de Veress visando à criação de pneumoperitônio artificial mediante insuflação de gás carbônico. A agulha utilizada foi de $12 \mathrm{~cm}$ de comprimento e $2 \mathrm{~mm}$ de diâmetro externo, de uso permanente, de marca Storz ${ }^{\circledR}$. A amostra constituiu-se de 69 mulheres (75,8\%) e 22 homens (24,2\%), com idade média de 47,92 anos (DP+-15,06, mediana=46, entre 16 e 86 anos). A média do Índice de Massa Corporal (IMC) foi 26,16 (+4.97), com mediana de 25,71, e amplitude entre 18,37 e 48,11 . Quarenta pacientes (44\%) foram considerado saudáveis pelo IMC (<25), enquanto que 34 (37.4\%) tinham sobrepeso (IMC entre 25 e 30), e 17 (18.7\%) eram obesos (IMC >30). Sessenta pacientes (65.9\%) tinham operação abdominal prévia. Nenhum critério de exclusão foi adotado.

Os pacientes foram submetidos à anestesia geral com intubação orotraqueal e ventilação controlada 
mecânica. Receberam previamente 0,1 mg/Kg de midazolam 30 minutos antes do ato anestésico. A indução anestésica foi realizada com $2 \mathrm{mg} / \mathrm{Kg}$ de propofol e 0,5 $\mathrm{mcg} / \mathrm{Kg}$ de fentanil, e a curarização com 0,5 mg/Kg de atracúrio. Logo após a intubação foi introduzida uma sonda orogástrica e aspirado o conteúdo do estômago.

Em decúbito dorsal horizontal, e com proclive de $30^{\circ}$. realizou-se a técnica de punção no hipocôndrio esquerdo. Fez-se incisão de $2 \mathrm{~mm}$ na pele ao nível do rebordo costal, a cerca de $8 \mathrm{~cm}$ da linha mediana, pela qual foi introduzida uma agulha perpendicularmente à parede abdominal anterior (Figura 1).

Após a punção foram utilizadas cinco provas para testar o posicionamento da ponta da agulha no interior da cavidade peritoneal (Figura 2).

Os testes obedeceram à seguinte sequência: prova da aspiração - PA, da resistência à infusão - Pres, da recuperação do líquido infundido - Prec, prova do gotejamento PG, e a prova da pressão intraperitoneal inicial (PPII) (Figura 2).

A prova da aspiração (PA) consistiu na aspiração com seringa de $10 \mathrm{ml}$ contendo $5 \mathrm{ml}$ de solução salina através da agulha de Veress, a qual era considerada positiva quando qualquer tipo de material se fazia presente na seringa e rotulada como negativa quando nenhum tipo de material lá existisse

Prova da resistência à infusão (Pres): injeção de $5 \mathrm{ml}$ de solução fisiológica através da agulha, verificandose moderada resistência ao fluir do líquido (prova positiva) ou, ao contrário, constatando-se importante aumento dessa resistência (prova negativa).

Prova da recuperação do líquido infundido (Prec): após a infusão de $5 \mathrm{ml}$ de solução fisiológica, aspirava-se a seringa, considerando-se positiva a prova

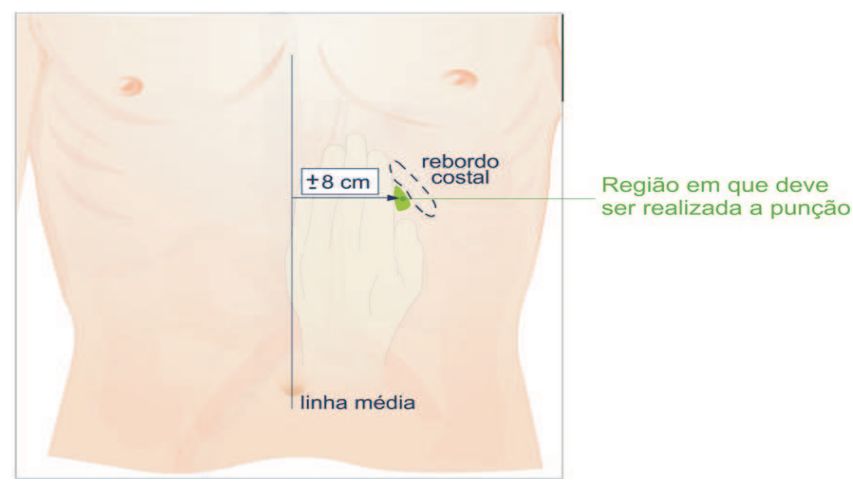

Figura 1 - Esquema representativo da localização da punção com agulha de Veress no hipocôndrio esquerdo, junto ao rebordo costal, a $8 \mathrm{~cm}$ da linha mediana do abdome. Observa-se o ponto em que deve ser realizada a punção, notando-se o distanciamento dos grandes vasos retroperitoneais (aorta abdominal, veia cava inferior, artérias e veias ilíacas primitivas) e dos vasos calibrosos da parede abdominal superior (artéria e veia epigástrica superior esquerda). quando não se recuperava o líquido infundido ou negativa quando todo ou parte do líquido infundido era recuperado.

Prova do gotejamento (PG): após gotejamento no reservatório da agulha, observava-se o desaparecimento imediato das gotas (prova positiva) ou, ao contrário, o acúmulo de líquido no reservatório (prova negativa).

Prova da pressão intraperitoneal inicial (PPII): era considerada positiva (agulha em posição adequada no interior da cavidade peritoneal e com orifício de saída de gás livre de obstrução) caso a pressão fosse igual ou menor que $8 \mathrm{mmHg}$ nos primeiros 10 segundos, e considerada negativa (agulha em posição inadequada ou com orifício obstruído) se a pressão fosse maior que esse valor e assim permanecesse por 10 segundos.

As Pres, Prec e PG foram realizadas e registradas conforme o protocolo pré-determinado, uma após a outra, quer se mostrassem positivas ou negativas. Após as provas, o insuflador foi regulado para emitir um fluxo de 1,2 l/min e a pressão intraperitoneal máxima foi programada para atingir $12 \mathrm{mmHg}$. Após a mangueira do insuflador ser conectada à agulha e após a manobra conhecida como "shaking" (pequena sacudidela para livrá-la de embaraços nos omentos) este era acionado, sendo então realizada a PPII. Caso a PPII fosse acima de $8 \mathrm{mmHg}$ e assim se mantivesse, era considerada então negativa, o procedimento era rotulado como fracasso e a agulha de Veress retirada e todo o procedimento reiniciado.

Com a PPII positiva, prosseguia-se com a insuflação de gás carbônico até a pressão atingir a marca de $12 \mathrm{mmHg}$, anotando-se o procedimento como bem sucedido após a introdução do trocarte e da óptica laparoscópica no interior da cavidade peritoneal.

A sensibilidade (S) das provas realizadas foi definida como a proporção dos pacientes comprovadamente portadores da condição investigada capazes de serem detectados pelas provas, segundo a fórmula: $\mathrm{S}=$ [verdadeiro-positivos/ (verdadeiro-positivos + falso-negativos)] $\mathrm{x}$ $100 \%$.

A verificação de que o paciente era de fato portador da condição investigada foi conferida pela efetiva criação do pneumoperitônio artificial, diagnosticado por visão direta através de óptica laparoscópica introduzida na cavidade peritoneal.

A especificidade (E) das provas foi definida como sendo a proporção dos pacientes sem a condição que foram corretamente diagnosticados como tal mediante a realização da prova, segundo a fórmula: $\mathrm{E}=$ [verdadeiro-negativos/(verdadeiro-negativos + falso-positivos)] $\times 100 \%$.

A verificação de que o paciente não era de fato portador da condição foi conferida pela impossibilidade efetiva de se insuflar a cavidade peritoneal. 


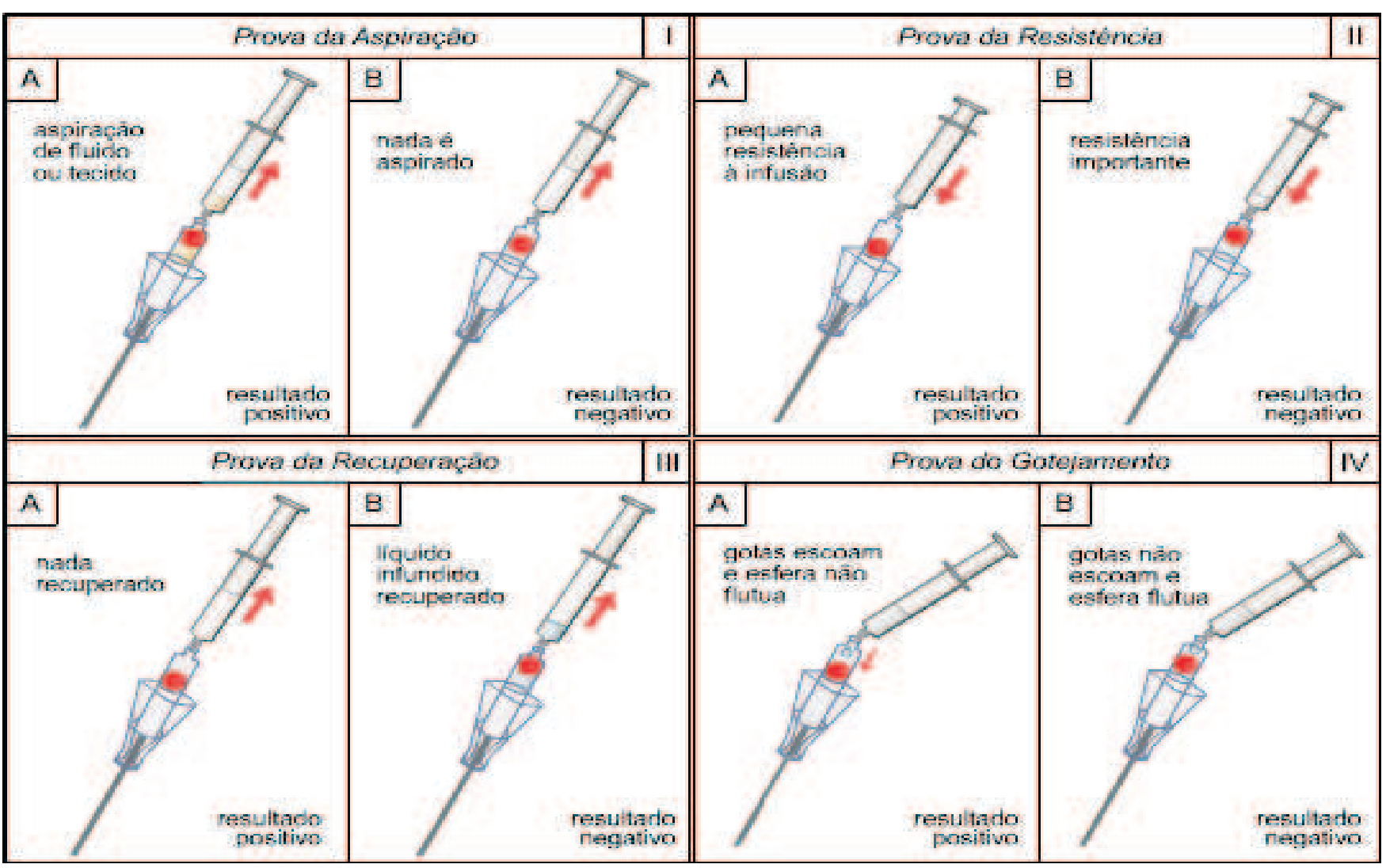

Figura 2 - Provas de posicionamento da ponta da agulha de Veress - Prova da aspiração (PA): aspiração com seringa de 5 ml através da agulha de Veress. Era considerada positiva quando qualquer tipo de material se fazia presente na seringa (I-A), e negativa quando nenhum tipo de material se fazia presente na seringa (I-B). Prova da resistência (Pres): injeção se 5 ml de solução fisiológica através da agulha, verificando-se moderada resistência ao fluir do líquido (II-A: prova positiva) ou, ao contrário, constatando-se aumento dessa resistência (II-B: prova negativa). Prova de recuperação (Prec): após a infusão de $5 \mathrm{ml}$ de solução fisiológica, aspira-se a seringa, considerando-se positiva a prova quando não se recuperava o líquido infundido (III-A), ou negativa quando todo ou parte do líquido infundido era recuperado (III-B). Prova do gotejamento (PG): após gotejamento no reservatório da agulha, observava-se o desaparecimento imediato das gotas (IV-A: prova positiva) ou, ao contrário, constatava-se o acúmulo de líquido no reservatório, com subida permanente da esfera da agulha (IV-B: prova negativa).

Foi considerado como o valor preditivo positivo (VPP) a probabilidade da agulha estar bem posicionada entre os resultados positivos de uma prova. O valor preditivo negativo (VPN) foi a probabilidade da agulha estar de fato mal posicionada entre os resultados negativos de uma prova. Ambos os valores prestaram-se para a avaliação da confiabilidade dos resultados das provas e foram calculados mediante as seguintes equações: VPP = [verdadeiropositivos/(verdadeiro-positivos + falso-positivos)] x 100\%; VPN = [verdadeiro-negativos/(verdadeiro-negativos + falso-negativos)] $\times 100 \%$.

Estudou-se a existência de correlação entre as variáveis pressão intraperitoneal e volume injetado nos pacientes do grupo como um todo, dentro de um limite de confiança de $95 \%$.

As variáveis qualitativas foram representadas por frequência absoluta e relativa e as quantitativas por média, desvio-padrão e valores mínimos e máximos. A equivalência ou não entre os grupos de estudo foi estabelecida pelo estudo da ocorrência ou não da sobreposição dos intervalos de confiança $\left(\mathrm{IC}_{95 \%}\right)$.

\section{RESULTADOS}

Não ocorreram complicações nas punções realizadas nesta pesquisa.

Houve 13 fracassos de estabelecer o pneumoperitônio na primeira tentativa de punção. Esses fracassos foram todos detectados pela prova de pressão intraperitoneal inicial (PPII). Nenhuma outra prova teve essa eficiência de 100\% demonstrada pela PPII na detecção dos fracassos realmente ocorridos (Tabela 1).

De uma maneira geral, a sensibilidade (S), a especificidade (E), o valor preditivo positivo (VPP) e o valor preditivo negativo (VPN) das provas de posicionamento intraperitoneal da ponta da agulha de Veress utilizadas nesta pesquisa foram os seguintes: teste de aspiração: $S=$ não aplicável; $E=100 \%$; VPP=não aplicável; VPN=100\%; teste de injeção: $S=100 \%$; $\mathrm{E}=0 \%$; VPP=85.71\%; VPN=não aplicável; teste de recuperação: $S=100 \% ; E=53.84 \% ; V P P=92.85 \%$; VPN $=100 \%$; teste do gotejamento: $S=100 \%$; $E=61.53 \%$; VPP $=93.97 \%$; VPN=100\%; teste da pres- 
são intraperitoneal inicial: $S=100 \% ; S=100 \%$; VPP $=100 \%$; VPN=100\% (Tabela 2).

\section{DISCUSSÃO}

A validade das provas para a localização da ponta da agulha de Veress na criação do pneumoperitônio já foi bem demonstrada ${ }^{18}$. Um estudo de comparação entre as duas técnicas de punção (umbilical versus hipocôndrio esquerdo) com nível suficiente de evidência, necessita de amostragem superior a 100.000 pacientes ${ }^{19}$ para detectar a redução de acidentes (prevalência de 0,05\% a 0,5\%) e complicações maiores. Dessa forma, o aprimoramento dos métodos de identificação do posicionamento da agulha e de lesões, mediante as provas de confirmação após a punção e durante a produção do pneumoperitônio, passa a ser de capital importância ${ }^{20}$. Também é útil a adoção de parâmetros de volume de gás injetado em cada momento e a pressão intraperitoneal resultante durante a insuflação para assegurar a locação correta do gás no interior da cavidade peritonea| ${ }^{21}$. Também foi demonstrado que a evolução da criação do pneumoperitônio se dá tão bem com a punção alternativa no hipocôndrio esquerdo como com a clássica, na linha mediana do abdome ${ }^{17}$.

Reitere-se que para comparar o risco desta punção com o risco da punção na linha mediana do abdome ao nível da cicatriz umbilical $(0,05$ a $1,8 \%)$, seria necessário um estudo prospectivo interessando 100.000 pacientes ${ }^{19}$. Entretanto, a maior segurança da punção do hipocôndrio esquerdo em comparação à punção clássica na cicatriz umbilical pode apenas ser deduzida a partir de considerações a respeito das relações topográficas das estruturas em risco, com vistas especiais para os grandes vasos retroperitoneais da linha median $a^{9}$ e para a topografia preferencial na linha mediana das aderências peritoneais. Há relatos de menor risco de lesão iatrogênica ${ }^{12-15}$ quando a punção é efetuada no hipocôndrio esquerdo, pelo fato desta situação ser fora da linha média, onde há maior chance de ocorrer lesões dos grandes vasos retroperitoneais ${ }^{12-13}$

Porém,na punção no hipocôndrio esquerdo devese ter em conta eventuais lesões dos vasos epigástricos superiores e das estruturas imediatamente posteriores à parede abdominal anterior no local da punção, como é o caso do corpo gástrico, cólon transverso e omento maior. Entretanto, os troncos e os ramos de maior calibre dos vasos epigástricos superiores nunca se situam a distância maior que oito centímetros da linha média do abdome, de forma que as punções realizadas além dessa distância evitam lesões dos referidos vasos ${ }^{21-23}$.

O valor das provas e parâmetros estabelecidos para avaliar a segurança no desenvolvimento do pneumoperitônio foi demonstrado, por Azevedo ${ }^{16-18}$, em pacientes sem operações abdominais prévias, passado de afecções inflamatórias intra-abdominais e com IMC abaixo de 30. O referido autor encontrou valores de sensibilidade, especificidade, predição positiva e negativa para cada prova realizada e demonstrou aquela que atinge $100 \%$ em todos estes quesitos: a Prova

Tabela 1 - Desempenho de cada uma das provas na detecção de fracassos na primeira tentativa de inserir a agulha de Veress na cavidade peritoneal.

\begin{tabular}{lccc}
\hline Prova $(n=91)$ & \multicolumn{3}{c}{ Provas negativas } \\
\cline { 2 - 4 } & Número & Proporção $(\%)$ & $95 \%$ IC \\
\hline Aspiração & 0 & 0 & - \\
Injeção & 0 & 0 & - \\
Recuperação & 6 & 6,59 & {$[0,013 ; 0,092]$} \\
Gotejamento & 5 & 5,49 & {$[0,011 ; 0,139]$} \\
Pressão intraperitoneal inicial & 13 & 14,29 & {$[0,028 ; 0,1806]$} \\
Tentativas falhas & 13 & 14,29 & {$[0,028 ; 0,1806]$} \\
\hline
\end{tabular}

IC = Intervalo de Confiança

Tabela 2 - Resultados das provas realizadas.

\begin{tabular}{|c|c|c|c|c|}
\hline Prova & Sensibilidade & Especificidade & VPP & VPN \\
\hline Aspiração & - & $100 \%$ & - & $100 \%$ \\
\hline Injeção & $100 \%$ & $0 \%$ & $85,71 \%$ & - \\
\hline Recuperação & $100 \%$ & $53 \%$ & $92,7 \%$ & $100 \%$ \\
\hline Gotejamento & $100 \%$ & $61,53 \%$ & $93,97 \%$ & $100 \%$ \\
\hline Pressão intraperitoneal inicial & $100 \%$ & $100 \%$ & $100 \%$ & $100 \%$ \\
\hline
\end{tabular}

VPP $=$ Valor Preditivo Positivo. VPN $=$ Valor Preditivo Negativo 
Pressão Intraperitoneal Inicial (PPII). Azevedo definiu como PPII não só a pressão medida pelo insuflador antes de iniciar-se a insuflação, mas também a constatada após os 10 segundos iniciais. Estabeleceu como limite superior de segurança o valor de $8 \mathrm{mmHg}$ - valores que permanecessem acima significariam posicionamento errôneo-, e encontrou valor médio inicial em torno de $4 \mathrm{mmHg}$.

Em conclusão, a punção com agulha de Veres no hipocôndrio esquerdo na criação do pneumoperitônio é segura e eficiente em amostragem ex- traída indiscriminadamente da população de pacientes submetidos à videolaparoscopia. As cinco provas testadas em conjunto são adequadas para orientar o cirurgião quanto ao posicionamento correto da ponta da agulha no início da insuflação a despeito de gênero, índice de massa corporal ou operações prévias.

Os autores agradecem à administração do Hospital Municipal José de Carvalho Florence, de São José dos Campos, São Paulo, e à Sociedade Paulista para o Desenvolvimento da Medicina (SPDM), o apoio financeiro e logístico para a realização desta pesquisa.

\section{A $B$ B S T R A $A$}

Objective: To assess the effectiveness of the Veress needle puncture in the left hypochondrium and the accuracy of the tests described for the intraperitoneal correct positioning of the tip of the Veress needle in an unselected population. Methods: Ninetyone patients consecutively scheduled for Videolaparoscopy had the abdominal wall punctured in the left hypochondrium. There were no exclusion criteria. The patients received general anesthesia and mechanical ventilation according to the protocol. After puncturing five tests were used to confirm the positioning of the needle tip within the peritoneal cavity: aspiration test - AT; resistance to infusion - Pres; recovery of the infused fluid - Prec, dripping test - DT, and test of initial intraperitoneal pressure - IIPP. The test results were compared with results from literature for groups with defined exclusion criteria. The results were used for calculating sensitivity (S) specificity (E), positive predictive value (PPV) and negative predictive value (NPV). Inferential statistical methods were used to analyze the findings. Results: There were 13 failures. AT had $E=100 \%$ and NPV 100\%. Pres had S $=100 \%$, $E=0 ; P P V=85.71 \% ;$ NPV does not apply. Prec: $S=100 \%, E=53.84 \%, P P V=92.85 \%, N P V=100 \% . D T: S=100 \%, E=61.53 \%$, $P P V=93.97 \%$ NPV $100 \%$. In IIPP, S, E, PPV and NPV were 100\%. Conclusion: The puncture in the left hypochondrium is effective and the performed tests guide the surgeon regardless of sex, BMI, or previous laparotomy.

Key words: Pneumoperitoneum, Artificial. Biopsy, Needle. Surgical Procedures, Minimally invasive. Surgical Procedure, Laparoscopy. Adverse effects, Laparoscopy.

\section{REFERENCIAS}

1. Shamiyeh A, Glaser $K$, Kratochwill $H$, Hörmandinger $K$, Fellner $F$, Wayand $\mathrm{WU}$, Zehetner.J. Lifting of the umbilicus for the installation of pneumoperitoneum with the Veress needle increases the distance to the retroperitoneal and intraperitoneal structures. Surg Endosc. 2009;23(2):313-7.

2. Catarci M, Carlini M, Gentileschi P, Santoro E. Major and minor injuries during the creation of pneumoperitoneum. A multicenter study on 12,919 cases. Surg Endosc. 2001;15(6):566-9.

3. Anaise D. Vascular and bowel injuries during laparoscopy. [monography of the Internet]. Disponível em http:// www.danaise.com/vascular_and_bowel_injuries_duri.htm

4. Wind J, Cremers JE, van Berge Henegouwen MI, Gouma DJ, Jansen FW, Bemelman WA. Medical liability insurance claims on entryrelated complications in laparoscopy. Surg Endosc. 2007:21(11):2094-9.

5. Neudecker J, Sauerland S, Neugebauer E, Bergamaschi R, Bonjer $\mathrm{HJ}$, Cuschieri A, Fuchs $\mathrm{KH}$, Jacobi $\mathrm{CH}$, Jansen FW, Koivusalo AM, Lacy A, McMahon MJ, Millat B, Schwenk W. The European Association for Endoscopic Surgery clinical practice guideline on the pneumoperitoneum for laparoscopic surgery. Surg Endosc. 2002;16(7):1121-43.

6. Veress J. Neues instrument zur Ausfuhrung von brust-oder bauchpunktionen und pneumothoraxbehandlung. Dtcsch Med Wochenshr. 1938;41(8):1480-1.

7. Molloy D, Kaloo PD, Cooper M, Nguyen TV. Laparoscopic entry: a literature review and analysis of techniques and complications of primary port entry. Aust N Z J Obstet Gynaecol. 2002;42(3):24654.
8. Tsaltas J, Pearce S, Lawrence A, Meads A, Mezzatesta J, Nicolson S. Safer laparoscopic trocar entry: it's all about pressure. Aust N Z J Obstet Gynaecol. 2004;44(4):349-50.

9. Pirró N, Ciampi D, Champsaur P, Di Marino V. The anatomical relationship of the iliocava junction to the lumbosacral spine and the aortic bifurcation. Surg Radiol Anat. 2005;27(2):137-41.

10. Roviaro GC, Varoli F, Saguatti L, Vergani C, Maciocco M, Scarduelli A. Major vascular injuries in laparoscopic surgery. Surg Endosc. 2002;16(8):1192-6.

11. Azevedo JL, Azevedo OC, Miyahira SA, Miguel GP, Becker OM Jr, Hypólito OH, Machado AC, Cardia W, Yamaguchi GA, Godinho L, Freire D, Almeida CE, Moreira CH, Freire DF. Injuries caused by Veress needle insertion for creation of pneumoperitoneum: a systematic literature review. Surg Endosc. 2009;23(7):1428-32.

12. Palmer R. Safety in laparoscopy. J Reprod Med. 1974;13(1):1-5.

13. Rohatgi A, Widdison AL. Left subcostal closed (Veress needle) aproach is a safe method for creating a pneumoperitoneum. J Laparoendosc Adv Surg Tech A. 2004;14(5):278-80.

14. Scwartz ML, Drew RL, Andersen JN. Induction of pneumoperitoneum in morbidly obese patients. Obes Surg. 2003:13(4):601-4

15. Hurd WW, Bude RO, DeLancey JO, Pearl ML. The relationship of the umbilicus to the aortic bifurcation: implications for laparoscopic technique. Obstet Gynecol. 1992;80(1):48-51.

16. Azevedo JLMC, Azevedo OC, Becker Júnior OM, Hypólito OHM, Machado ACCG, Freire DF. Veress needle insertion in the left hipochondrium in creation of the pneumoperitoneum: validation of the technique, value of tests and importance of intraperitoneal pressure and volume of gas injected during insufflations. Bras J Vídeo-Sur. 2008;1(1):20-8. 
17. Azevedo OC, Azevedo JL, Sorbello AA, Miguel GP, Guindalini RS, Godoy AC. Veress needle insertion in the left hypochondrium in creation of the pneumoperitoneum. Acta Cir Bras. 2006;21(5):296303.

18. Azevedo OC, Azevedo JL, Sorbello AA, Miguel GP, Wilson Júnior $J$, Godoy AC. Evaluation of tests performed to confirm the position of the Veress needle for creation of pneumoperitoneum in selected patients: a prospective clinical trial. Acta Cir Bras. 2006;21(6):38591.

19. Cakir T, Tuney D, Esmaeilzadem S, Aktan AO. Safe Veress needle insertion. J Hepatobiliary Pancreat Surg. 2006;13(3):225-7.

20. Fletcher RW, Fletcher SE. Risco: um olhar sobre o futuro. In: Fletcher RW, Fletcher SE. Epidemiologia clínica: elementos essenciais. 4. ed. Porto Alegre: Artmed; 2006. p. 98-115.

21. Azevedo JL, Azevedo OC, Sorbello AA, Becker OM, Hypólito O Freire D, Miyahira S, Guedes A, Azevedo GC. Intraperitoneal pressure and volume of gas injected as effective parameters of the correct position of the Veress needle during creation of pneumoperitoneum. J Laparoendosc Adv Surg Tech A 2009;19(6):731-4.

22. Chandler JG, Corson SL, Way LW. Three spectra of laparoscopic entry access injuries. J Am Coll Surg. 2001;192(4):478-90; discussion 490-1
23. Balzer KM, Witte $H$, Recknagel S, Kozianka J, Waleczek H. Anatomic guidelines for the prevention of abdominal wall hematoma induced by trocar placement. Surg Radiol Anat. $1999 ; 21(2): 87-9$

Recebido em 04/01/2010

Aceito para publicação em 28/02/2010

Conflito de interesse: nenhum

Fonte de financiamento: nenhuma

\section{Como citar este artigo:}

Becker Júnior OM, Azevedo JLMC, Azevedo OC, Hypólito OHM, Miyahira SA, Miguel GPS, Machado ACCG. Punção com agulha de veress no hipocôndrio esquerdo para a criação do pneumopertôneo: valor diagnóstico das provas de posicionamento da agulha em pacientes não selecionados. Rev Col Bras Cir. [periódico na Internet] 2011; 38(1). Disponível em URL: http://www.scielo.br/rcbc

\section{Endereço para correspondência:}

Prof. Dr. João Luiz Moreira Coutinho de Azevedo E-mail: jozevedo.dcir@epm.br 